\title{
Study on photochromic properties of metal dithizonate complexes
}

\section{doped PMMA film}

\author{
Shengwen $\mathrm{Qi}^{1,2,3, a}$, Xueping Cheng ${ }^{1, b}$ and Hongguang $L u^{4, c}$ \\ ${ }^{1}$ School of Mathematics, Physics and Information Science, Zhejiang Ocean University, Zhoushan \\ 316022, China \\ ${ }^{2}$ Key Laboratory of Oceanographic Big Data Mining \& Application of Zhejiang Province, Zhoushan, \\ Zhejiang 316022, China \\ ${ }^{3}$ Shandong Provincial Key Laboratory of Functional Macromolecular Biophysics, Dezhou 253023, \\ China \\ ${ }^{4}$ Affiliated Hospital, Guiyang Medical University, Guiyang 550002, China \\ a qi1963@163.com, ${ }^{b}$ xpcheng@163.com, ${ }^{c}$ hongguanglu@yahoo.com
}

Key words: mercury dithizonate, photochromic properties, z-scan

Abstract: The absorption spectra of trans and cis isomers of mercury dithizonate (MD) in doped polymethyl methacrylate (PMMA) film are measured, and the photochromic properties of the two isomers are analyzed individually. The experiments indicate that different isomers of MD can convert into each other when irradiated by light of proper wavelength. The nonlinear optical properties of MD are investigated by z-scan method, and the experimental data of nonlinear refractive index is obtained.

\section{Introduction}

The photochromism of metal-dithizonate complexes was discovered by Irving ${ }^{[1]}$ and Webb ${ }^{[2]}$ respectively in the $1950 \mathrm{~s}$. By the investigation of the mechanism of photochromism, Meriwether ${ }^{[3]}$ et al. proposed that the photochromism process was the isomerization reaction in the molecules of mercury dithizonate (MD), which included two processes: hydrogen transfer between N-N and cis-trans isomerization of $\mathrm{C}-\mathrm{H}$ double-bond. Wu Shikang ${ }^{[4]}$ investigated the dynamics of MD in macromolecules and found the processes of S-Hg bond's breakdown and the photon's transfer were the key to photochromism, while isomerization seldom occured. Wang Duo et al. ${ }^{[5]}$ explored the return process of MD in polymer, and concluded that the photochromic mechanics was a bimolecular process, i.e. transfer of $\mathrm{H}$ between molecules, instead of transfer process of hydrogen in one molecule. Metal dithizonate complexes have both photochromic properties at a wide range of wavelength and significant nonlinear optical properties, ${ }^{[6,12]}$ so they have potential applications in many fields. We will study the properties of their isomerization of MD sample by z-scan method in this paper.

\section{The preparation of the sample of MD and its absorption spectra of two isomers}

The structure and isomers of MD are shown in Fig.1. The preparation of MD polymer film is as follows: MD and PMMA dissolves in chloroform respectively, the solution of MD $\left(10^{-4} \mathrm{~mol} / \mathrm{L}\right)$ and the solution of PMMA (5\% wt) are mixed together completely, then the mixed solution is spread on a clean glass plate uniformly and dried at room-temperature for $24 \mathrm{~h}$. The thickness of the film is 
about $40 \mu m$, and the concentration of MD

in PMMA medium is $5.89 \times 10^{-3} \mathrm{~mol} / \mathrm{L}$, which is measured by the method of standard working curve.

The absorption spectrum of MD polymer film is measured in the dark, then the MD polymer film is irradiated by a He-Cd laser $\left(443 \mathrm{~nm}, 5 \mathrm{~mW} / \mathrm{cm}^{2}\right)$ for about $10 \mathrm{~s}$, and as a result, most of the MD molecules isomerize from trans to cis and

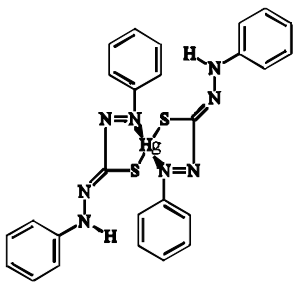

a

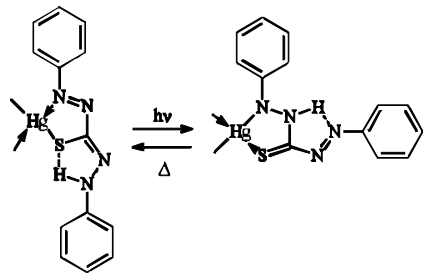

b

Fig. 1 Molecular structure and isomers of MD

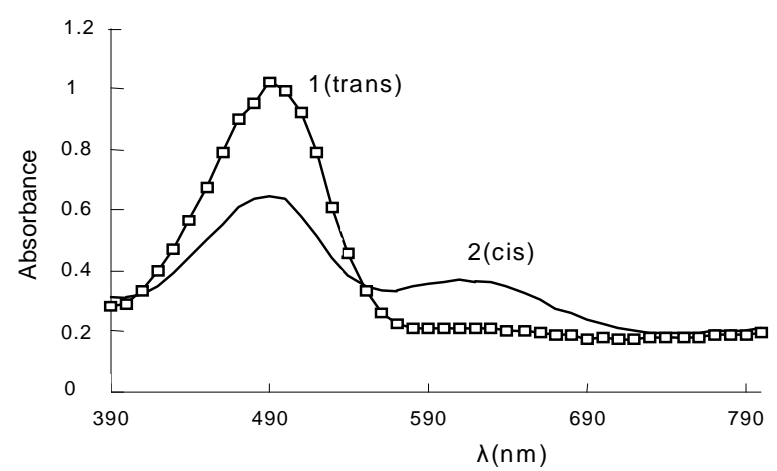

Fig. 2 Absorption spectra of trans and cis isomers of MD doped PMMA film $490 \mathrm{~nm}$ increases. Accordingly, the contribution of the molecules in trans to absorption spectrum decreases, and the absorption peak corresponding to trans decreases too. On the contrary, the number of the molecules in cis increases, and the contribution of the molecules in cis to absorption spectrum increases, so the corresponding absorption peak becomes higher. Comparing Curve 1 with curve 2, we can conclude that the absorption peaks at $490 \mathrm{~nm}$ and $630 \mathrm{~nm}$ refer to trans isomer and cis isomer respectively.

After irradiated by a He-Cd laser beam, the sample of MD can keep blue for a couple of days. This property indicates that MD polymer film can be used as an optical storage material. Affected by the irradiation of He-Ne laser $(\lambda=633 \mathrm{~nm})$, the cis isomer isomerizes to trans isomer, so the blue color of the sample disappears and the film returns to the original brown color. This means that the information stored in the sample can be erased. So MD polymer film can be used as erasable optical storage medium.

\section{Experiments of z-scan}

Z-scan method, put forward by M. Sheik-Bahae in 1989, is a simple and sensitive technique for determining the intensity dependent index of refraction $n_{2}$ of cubical nonlinear optical materials. ${ }^{[13]}$ In the experimental setup shown in Fig.3, a Gaussian beam focuses on to the thin sample and undergoes a phase and amplitude distortion during

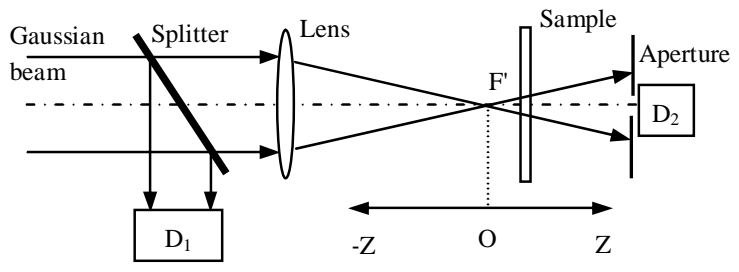

Fig. 3 Schematic diagram of z-scan 
its propagation. An aperture and a photodetector $\mathrm{D}_{2}$ are centered at the beam and located at the far field to record the power passing the aperture. Photodetector $D_{1}$ is used to detect part of the incident power and eliminate the effect of the fluctuation of incident power on measured results. Because of the lensing effect caused by nonlinear refraction, the beam transmitting the sample will diverge for negative nonlinear materials or will focus for positive nonlinear materials. When the sample is scanned around the waist of the focused beam along the z-axis, the measured transmitted power will change with the distance that the sample is from the waist position. A typical peak-valley transmittance curve is obtained and then the nonlinear refractive index can be inferred.

In our experiments ${ }^{[13,14]}$, the condition $\pi \omega^{2} / \lambda>L$ is always satisfied for an optical thin sample, so $\Delta n$ and $n_{2}$ can be calculated by ${ }^{[15]}$

$$
\begin{aligned}
& \Delta n=\frac{\lambda}{2 \pi} \frac{\alpha}{1-e^{-\alpha L}} \frac{\Delta T_{P-V}}{0.406(1-s)^{0.25}} \\
& n_{2}=\frac{\pi \omega^{2}}{2 P} \Delta n
\end{aligned}
$$

where $n_{2}$ is effective index of refraction of cubical nonlinear optical materials, $\Delta n$ is refractive index change, $\lambda$ is the free space wavelength of the illumination beam, $\alpha$ is the effective absorption coefficient, $L$ is the thickness of the sample, $\Delta T_{\mathrm{P}-\mathrm{V}}$ is the normalized transmittance difference between maximum and minimum transmittances, $s$ is the linear transmittance, $P$ is the power of the Gaussian beam, and $\omega$ is the waist radius of the illumination beam inside the film.

Using He-Ne laser $(\lambda=633 \mathrm{~nm})$ as a probe beam, the measured results of z-scan are shown in Fig. 4. The curves in Fig. 4 is normalized to its value when the sample is located in the focus position and the effect of intensity-dependent transmittance of the sample on the measured results was eliminated. From Fig.4 we can get $\Delta T \mathrm{p}-\mathrm{v}=0.2$. Since $L=0.040 \mathrm{~mm}$, $P=4 m W, s=0.5, \omega=0.037 \mathrm{~mm}$ and $\alpha=12.2 \mathrm{~mm}^{-1}$, we obtained $\Delta n=-0.0011$ and $n_{2}=-6.04 \times 10^{-10} \mathrm{~m}^{2} / W$.

Similarly, for the probe beam of $\lambda=535 \mathrm{~nm}$, and the corrected results of z-scan are shown in Fig.5 respectively. From Fig.5 we got $\Delta T \mathrm{p}-\mathrm{v}=0.7$. Considering $L=0.040 \mathrm{~mm}, P=3 \mathrm{~mW}, s=0.5, \omega=0.060 \mathrm{~mm}$ and $\alpha=12.2 \mathrm{~mm}^{-1}$, we obtained $\Delta n=-0.0034$ and $n_{2}=-7.06 \times 10^{-9} \mathrm{~m}^{2} / W$.

\section{Conclusion}

The nonlinear optical properties of MD doped PMMA film were measured and the refractive index change and the intensity dependent index of refraction are obtained by z-can method at the wavelengths of $633 \mathrm{~nm}$ and $535 \mathrm{~nm}$ respectively. The results indicate that MD has larger optical nonlinearity and wide responsive bandwidth:

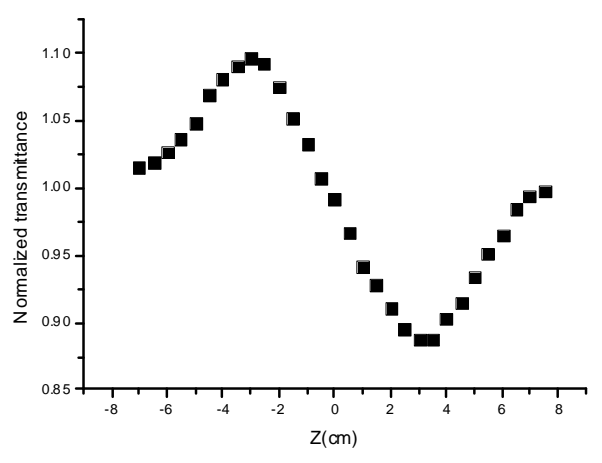

Fig. 4. Normalized z-scan transmittance of MD film with the 633-nm laser

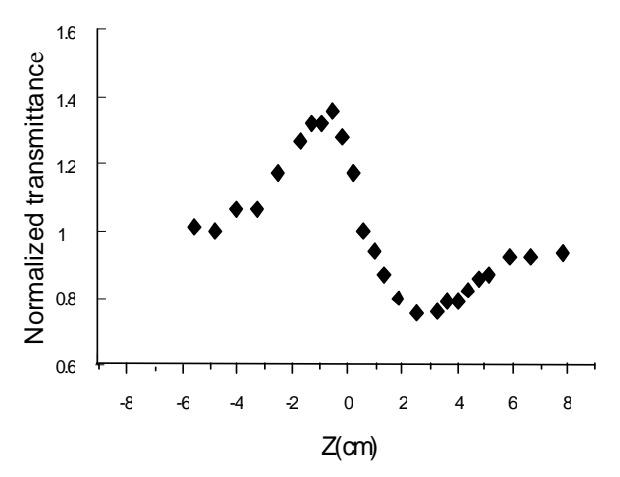

Fig. 5 Normalized z-scan transmittance of MD film with the 535-nm laser 
(1) $\mathrm{n}_{2}$ of MD sample has been determined to be in the range from $10^{-10}$ to $10^{-9} \mathrm{~m}^{2} / \mathrm{W}$, while other nonlinear materials, such as the poly (phenylacetylene) and poly (p-methoxyphenylacetylene), $n_{2}=6 \times 10^{-22} \mathrm{~m}^{2} / W$ and $n_{2}=1.1 \times 10^{-21} \mathrm{~m}^{2} / W$, respectively; ${ }^{[16]}$ the averaged $n_{2}$ values for crystals $\mathrm{LN}: \mathrm{Mg}$ and KTP are $2.0 \times 10^{-19}$ and $1.2 \times 10^{-19} \mathrm{~m}^{2} / W$, respectively; [17] for eosin in polymer film, $n_{2}=5.58 \times 10^{-16} \mathrm{~m}^{2} / W^{[18]}$

(2) For instance, the absorption spectrum of azobenzene doped PMMA ranges from 380 $\mathrm{mm}$ to $400 \mathrm{~nm} ;{ }^{[19]}$ the absorption spectrum of methyl red dye-doped PMMA film is from $400 \mathrm{~nm}$ to $550 \mathrm{~nm}^{[20]}$. Comparatively, the absorption spectra of MD range from $390 \mathrm{~nm}$ to $690 \mathrm{~nm}$, so that almost whole range of visible light can change the optical nonlinearity of MD.

\section{Acknowledgment}

This work is financially supported partly by the Natural Science Foundation of Shandong (ZR2014FL028), the National Natural Science Foundation of China (No.11505154, No.11305141) and the Natural Science Foundation of Zhejiang ( LQ16A010003) .

\section{References}

[1] Irving H, Andrew G, Risdon E J et al.: J Chem Soc Vol. 12(1949), p.541

[2] Webb J L A, Corwin A H, Sharp A G et al.: J Am Chem Soc Vol. 72(1950), p.91

[3] Meriwether L S, Breither E C, Sloan C L et al.: J Am Chem Soc Vol. 87(1965), p.4441

[4] Wu Shikang, Shen Yanqing: Functional Polymer Vol. 3(1990), p.216

[5] Wang Duo, Wu Qiang: Acta Sci Natu. Univ. Nank. Vol. 4(1987), p.96

[6] Gulam RM, Takahashia T, Ohga Y: PHY CHEM CHEM PHY Vol. 11(2009), p.5170

[7] Armelao L, Bandoli G, Barreca D et al.:APP ORGA CHEM Vol. 21(2007), p.246

[8] Mandal M, Basu S: APPLIED RADIATION AND ISOTOPES Vol. 67(2009), p.256

[9] Gurkan R, Savasci S, Ozkan MH et al.: J. ANAL. CHEM. Vol. 60 (2005), p. 800

[10] Yamaki SB, de Oliveira MG, Atvars TDZ: J. BRAZ. CHEM. SOC. Vol. 15(2004), p. 253

[11] El-Mosallamy EH, Mekewi MA: MAT. CHEM. \& PHY. Vol. 80(2003), p. 319

[12] Feneyrou P, Soyer F, Le Barny P etc.: PHOT. \& PHOT. SCI. Vol. 2(2003), p.195

[13] M. Sheik-Bahae, A. A. Said, and E. W. Van Stryland: Opt. Lett. Vol. 14(1989), p.955

[14] Q. W. Song and C. Zhang: Opt. Lett. Vol. 18 (1993), p. 775

[15] S. Qi, X. Yang, C. Zhang et al.: J. Modern Optics Vol. 51 (2004), p. 1671

[16] M. Falconieri, R. D. Amato, A. Furlani et al.: Synthetic Metals Vol. 124(2001), p. 217

[17] H.P. Li, C.H. Kan, Y.L. Lam et al.: Optical Materials Vol. 15(2001), p.234

[18] Haosheng Fei: Physics Vol. 23(1994), p.178

[19] Xu Xingsheng, Ming Hai, Wang Pei et al.: J. Opt. A: Pure Appl. Opt. Vol. 4(2002), p.L5

[20] Guangbin Wang, Fuxi Gan: Materials Letters Vol. 43(2000), p.6 